\title{
Applications of nanotechnology in food processing and food packaging: A review
}

\author{
Idrees Ahmed Wani
}

\section{Department of Food Science \& Technology, University of Kashmir, Srinagar, India, 190006 \\ Email: waniidrees@kashmiruniversity.ac.in}

Nanotechnology is a powerful interdisciplinary tool for the development of innovative products in food industry. Nano inside technology in its advanced form involves the encapsulation of different food additives within the carrier materials of higher stability. The targeted and site-specific delivery of several bioactive compounds like polyphenols and antioxidants in microcapsules of $3-800 \mu \mathrm{m}$ diameter is a promising feature of this technology. Literature reported the encapsulation of beta-carotene in sorbitan monostreate and triacylglycerol, nisin in soy and marine lecithin and the commercially available vitamins, fatty acids and probiotics in different carriers. Conversely, nano outside involves the packaging of processed and raw foods like fresh horticultural commodities. In the present review, the potential applications of nanomaterials in food packaging and food processing are reviewed. In food packaging, the use of nanocomposites as high barrier packaging materials, silver nanoparticles as potential antimicrobial agents and nanosensors as detectors of microbial contamination or adulteration in complex food matrix is commonly observed. Nano food processing is reported to eliminate the harmful chemicals and pathogens from the food with a profound impact on sensory and the nutritional characteristics. Such applications in food systems are speculated to improve the safety and the nutritional value of food products (Figure 1).

Keywords: Nanotechnology, nano inside, nanocomposite, encapsulation, nanosensor

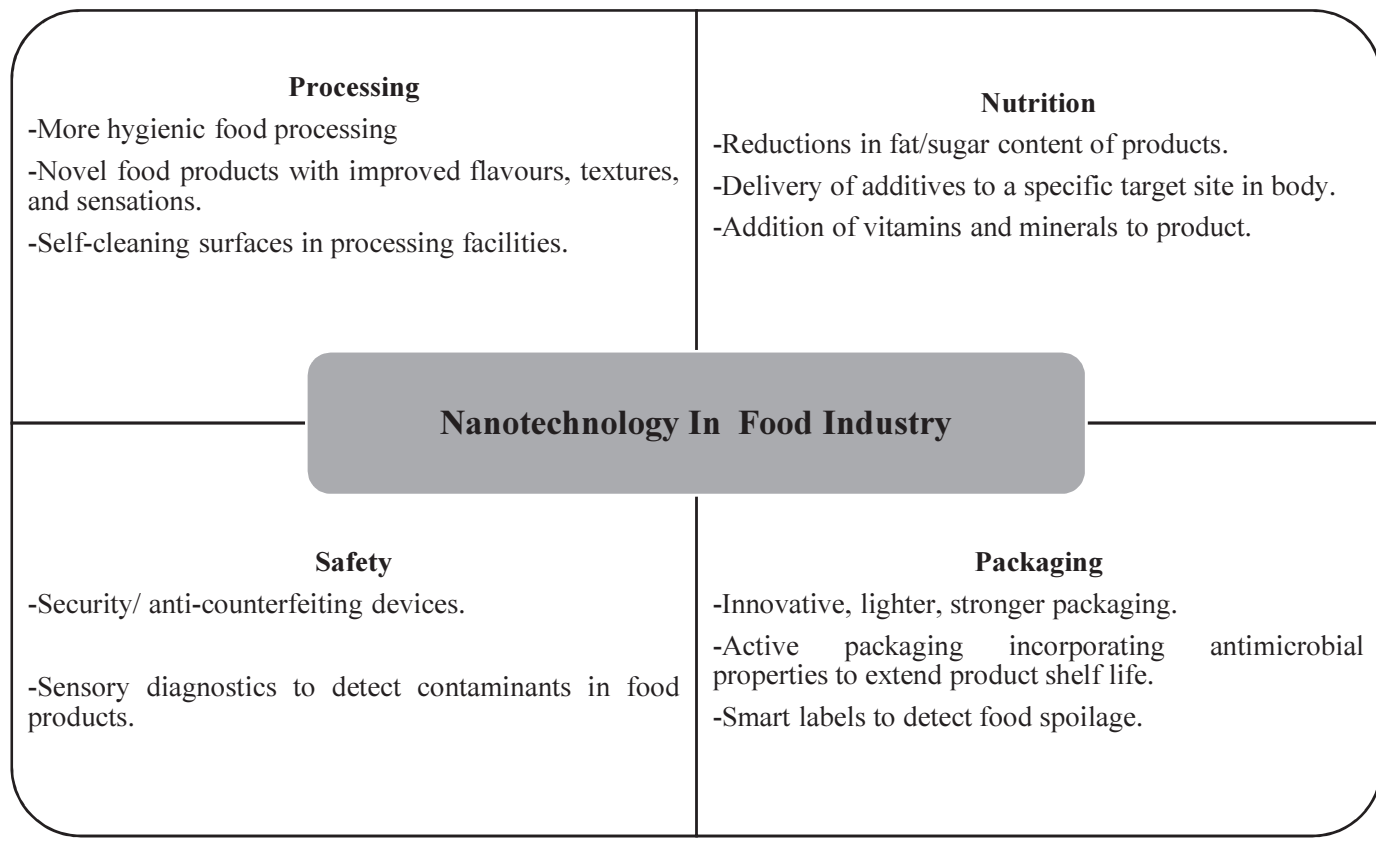

Figure 1: Schematic diagram of applications of nanotechnology in food technology 